\title{
Assessment of Foot Care Knowledge among Diabetic Amputees at National Orthopaedic Hospital Enugu
}

\author{
Osita Ede, Gabriel O. Eyichukwu, Emmanuel C. Iyidobi, Basil C. Nwachukwu \\ Department of Orthopaedics and Trauma, National Orthopaedic Hospital, Enugu, Nigeria \\ Email: dreciyidobi@yahoo.com
}

How to cite this paper: Ede, O., Eyichukwu, G.O., Iyidobi, E.C. and Nwachukwu, B.C. (2018) Assessment of Foot Care Knowledge among Diabetic Amputees at National Orthopaedic Hospital Enugu. Journal of Biosciences and Medicines, 6, 25-32. https://doi.org/10.4236/jbm.2018.64002

Received: March 12, 2018

Accepted: April 10, 2018

Published: April 13, 2018

Copyright (c) 2018 by authors and Scientific Research Publishing Inc. This work is licensed under the Creative Commons Attribution International License (CC BY 4.0).

http://creativecommons.org/licenses/by/4.0/

(c) (i) Open Access

\begin{abstract}
Diabetics with unilateral lower extremity amputation (LEA) have an increased risk of contralateral re-amputation. Foot care knowledge and practice has been shown to reduce the incidence of amputation. The study aims to assess the level of foot care knowledge among diabetics with unilateral LEA. Method: A questionnaire based study involving diabetics with unilateral LEA from August 2015 to August 2017. Demographic data and level of foot care knowledge were assessed. Associations between age, gender, educational level, prior amputation and duration of diabetes with level of foot care knowledge were investigated. The presence of peripheral neuropathy in the contralateral leg was also assessed. Results: A total of 64 patients had unilateral LEA within this period, but only 42 patients completed the study. Twenty-four, 24 (57.1\%) were males while $18(42.9 \%)$ were females. Mean age was 58.3 years (SD = 12.0). Fifteen, 15 (35.7\%) had secondary, 15 (35.7\%) had tertiary education, while $3(7.1 \%)$ and $9(21.4 \%)$ had none and primary education respectively. Below knee amputation (BKA) was the commonest (78.6\%), and $35.7 \%$ have had a prior amputation. Peripheral neuropathy was present in $71.4 \%$ of cases. No participant had a good level of foot care knowledge, while $13(31 \%)$ have a fair knowledge and 29 (69\%) have poor knowledge. Only 18 (42\%) said they have been taught on foot care. Only duration of diabetes and educational level were significantly associated with level of foot care knowledge. Conclusion: Diabetics with unilateral LEA have poor knowledge of foot care and a high prevalence of peripheral neuropathy. Efforts at education of these susceptible cohorts should be intensified.
\end{abstract}

\section{Keywords}

Diabetics, Amputation, Foot Care, Knowledge 


\section{Introduction}

Limb loss is a feared complication of diabetes mellitus. About $50 \%$ of diabetics with unilateral LEA will lose the contralateral limb in 4 years [1]. The burden of amputation is enormous, ranging from loss of job and income, to depression and reduced quality of life [2]. The mortality rate is also lower in patients with amputations compared to non-amputees [1] [3]. Peripheral neuropathy, peripheral vascular disease, and foot trauma, alone or in combination, can lead to ulcerations. This is frequently compounded by infection which can progress to gangrene and amputation.

The benefits of foot care knowledge and practice in reducing the incidence of amputations in diabetics have been shown [4] [5] [6]. This is even more important in developing countries such as Nigeria, where poverty abounds and patients seek medical intervention late. It is projected that Sub-Saharan African countries will experience the greatest rise in the prevalence of diabetes mellitus in the next decade [6]. Therefore simple preventive measures such as foot care practices will save considerable cost and mitigate the morbidity associated with bilateral lower extremity amputations.

It is generally assumed, and rightly so, that diabetics, who had amputations in hospitals, should receive some form of foot care education before discharge, in order to prevent a re-amputation. In the absence of a dedicated foot clinic and podiatrists in our clime, this responsibility falls on the managing health team (Doctors and Nurses).

Therefore, this study aims to assess how well diabetics with unilateral LEA are being educated on foot care. We also investigated the relationship between age, gender, educational level, prior amputation and duration of diabetes with level of foot care knowledge. Finally, the presence of peripheral neuropathy in the contralateral limb was also assessed.

\section{Materials and Methods}

This was a cross-sectional study done on diabetic patients with unilateral LEA at National Orthopaedic Hospital, Enugu (NOHE), from August 2015 to August 2017. NOHE is a regional orthopaedic and trauma center which serves the South-East and South-South geo-political zones of the country. A total of 64 patients had unilateral LEA within this period. A pilot study done by the researchers showed a point prevalence of LEA of $4 \%$ among diabetics admitted in our hospital. This gives a minimum sample size of 59 patients at $5 \% \alpha$-level and $80 \%$ power. A convenient sampling to include all patients was used. Inclusion criteria were all diabetic patients with unilateral LEA who gave consent. Exclusion criteria were non-diabetic related amputation, bilateral amputations, patients who rejected consent. Ethical approval was obtained from the hospital ethical committee and the patients.

A 15 item questionnaire on foot care adapted from previously validated questionnaire [5] [6] [7], and tested for internal reliability, with a Cronbach alpha 
value of 0.62 , was administered by the researchers to the participants. Plain, simple language was used to enhance understanding of the questions by participants. Participants who had been discharged were interviewed over the phone, and clinic appointments were scheduled to test for peripheral neuropathy. Patients who failed to keep to the appointments were dropped from the study. Peripheral neuropathy was assessed with the $128-\mathrm{Hz}$ vibrating tuning fork applied on the $1^{\text {st }}$ metatarsophalangeal joint, and compared with that of the researcher.

The questions were worded such that " 0 " meant no knowledge while " 1 " meant appropriate knowledge. The total score for each participant was thus obtained. A total score of 13 to 15 was taken as good knowledge of foot care, 10 to 12 as fair knowledge, while a score of less than 10 was taken as poor foot care knowledge. The ages of the participant were categorized, into young (less than $45)$, middle age (45 to 65 ) and old age (greater than 65 years).

Chi-square test of independence was used to assess the relationship between gender and prior amputation with foot care knowledge, while correlation was done for age, duration of diabetes and level of education. A $p$ value of less than 0.05 was considered significant.

\section{Results}

Only 42 patients completed the study. Six (6) patients were unreachable by phone, while 10 patients defaulted from the scheduled clinic appointments. Four (4) patients were reported dead, while 2 patients refused consent. Twenty-four, $24(57.1 \%)$ were males while 18 (42.9\%) were females. Figure 1 showed the age and sex distribution of the patients. Mean age was 58.3 years $(\mathrm{SD}=12.0)$. Fifteen, 15 (35.7\%) had secondary, 15 (35.7\%) had tertiary education, while 3 $(7.1 \%)$ and $9(21.4 \%)$ had none and primary education respectively. There were

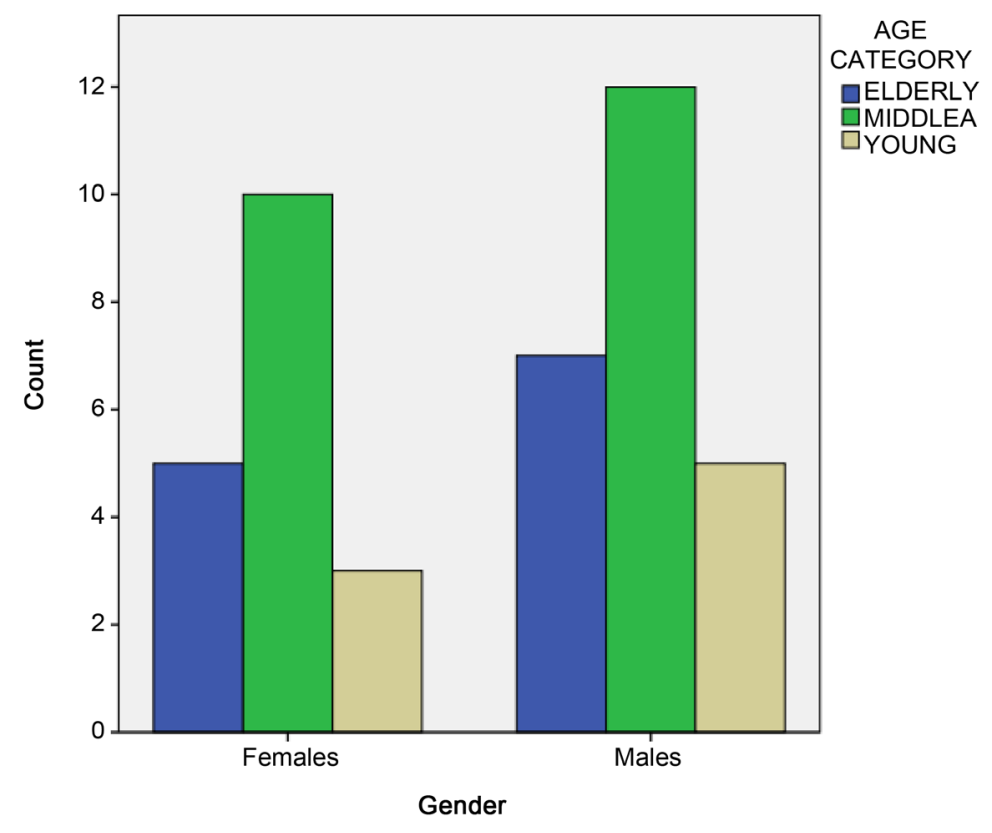

Figure 1. Clustered bar chart showing gender and age category distribution. 
$6(14.3 \%)$ ray amputation, $33(78.6 \%)$ below knee amputations, and $3(7.1 \%)$ above knee amputations. The commonest limb involved is the right limb, 36 $(85.7 \%)$.

Fifteen, 15 patients $(35.7 \%)$ have had a prior amputation of the affected limb. Peripheral neuropathy was present in the contralateral limb in 30 (71.4\%) of the participants. Twelve patients, 12 (28.6\%) were elderly (greater than 65 years), 22 (52.4\%) were middle aged while $8(19.0 \%)$ were young. Table 1 illustrated the socio-demographic and clinical characteristics of the patients.

Thirty-three, 33 patients (78.6\%) know that diabetics are at high risk of foot ulcer and amputation. Twenty-one, 21 patients (50\%) know that they should inspect their feet daily. Thirty, 30 (71.4\%) know they should see a doctor if they develop wound on their feet. Eighteen, 18 (42.9\%) know they should trim their nails straight across with a clipper. Twenty-seven, 27 (64.3\%) know they should keep their feet supple with moisturizers, but only $9(21.4 \%)$ know they should keep in-between their toes dry.

Twenty-four, 24 (57.1\%) know they should never walk barefoot even inside their homes. Twenty-four, 24 (57.1\%) know they shouldn't feel tight in their in their shoes. Eighteen, 18 (42.9\%) know they shouldn't wear pointed shoes or high heels. Twenty-one (50\%) know they should always inspect inside their shoes before wearing them. Twenty-four, 24 (57.1\%) know they should never wear shoes without socks, while 9 (21.4\%) know they should never use hot water bottle on their feet.

Eighteen, 18 (42.9\%) know they should never use their feet to check the temperature of an object. Twenty-four, 24 (57.1\%) know they should have their feet

Table 1. Socio-demographic and clinical characteristics of patients.

\begin{tabular}{cc}
\hline Characteristics & Proportion (n) \\
\hline Age (yrs) & 8 \\
$<45$ & 22 \\
$45-65$ & 12 \\
$>65$ & \\
Gender & 24 \\
M & 18 \\
F & \\
Type of amputation & 3 \\
Above knee & 33 \\
Below knee & 6 \\
Ray & \\
Educational level & \\
None & 3 \\
Primary & 7 \\
Secondary & 15 \\
Tertiary & 15 \\
Prior amputation & \\
Yes & 15 \\
No & 27 \\
\hline
\end{tabular}


examined by a professional at least once a year. Eighteen, 18 (42.9\%) said they have received at least some form of foot care education. Overall, 29 (69\%) have poor foot care knowledge, 13 (31\%) have fair knowledge while none (0\%) have good knowledge score. This is shown in Figure 2.

There were no significant effects of age $(r=0.11, p=0.50)$, gender $(p=0.34)$, and prior amputation (0.49) with level of foot care knowledge. However, duration of diabetes showed a strong correlation with foot care knowledge (rho = $0.56, \mathrm{p}<0.001)$. Educational level showed moderate correlation with level of foot care knowledge ( $\mathrm{rho}=0.45, \mathrm{p}=0.03$ ), see Table 2 . Table 3 is a list of the questions on foot care practices that was used in this study.

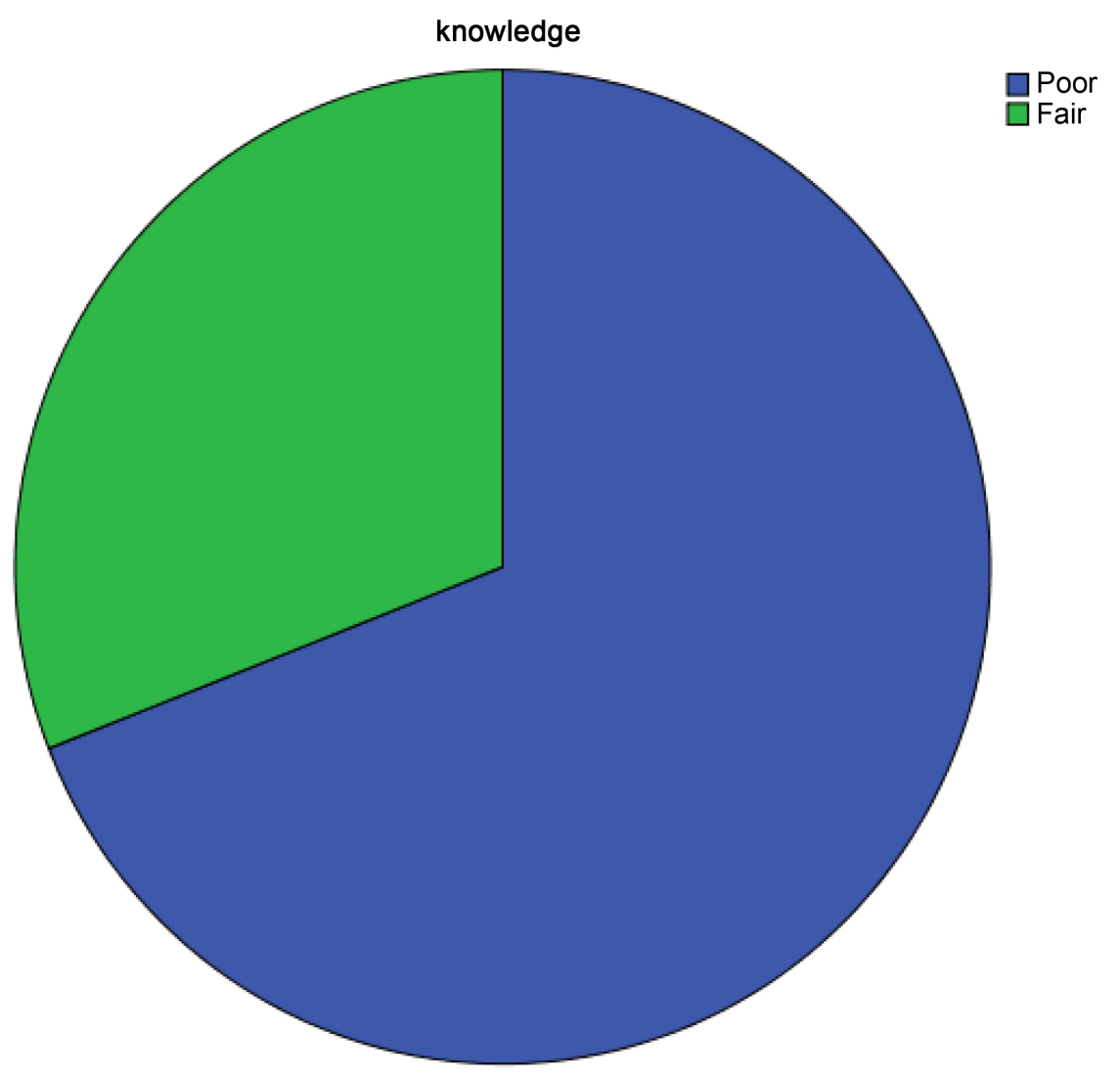

Figure 2. Pie chart showing distribution of knowledge scores.

Table 2. Effects of variables on level of foot care knowledge among diabetic patients.

\begin{tabular}{cc}
\hline Variable & p-value \\
\hline Age & 0.50 \\
Gender & 0.34 \\
Educational level & $0.03^{*}$ \\
Prior amputation & 0.49 \\
Duration of diabetes & $0.00^{*}$ \\
\hline
\end{tabular}

* = significant value. 
Table 3. Distribution of answers to questions related to level of foot care knowledge.

\begin{tabular}{ccc}
\hline Questions & Yes (\%) & No (\%) \\
\hline Diabetics are at high risk of foot ulcers and amputations? & 78.6 & 21.4 \\
Diabetics should inspect their feet daily? & 50 & 50 \\
Diabetics should see a doctor if they develop wound on their feet? & 71.4 & 28.6 \\
Diabetics should trim their nails straight across with a clipper? & 42.9 & 57.1 \\
Diabetics should keep their feet supple with a moisturizer? & 64.3 & 35.7 \\
Diabetics should keep in-between toes dry? & 21.4 & 78.6 \\
Diabetics should never walk barefoot even inside their homes? & 57.1 & 42.9 \\
Diabetics should never feel tight in their shoes? & 57.1 & 42.9 \\
Diabetics shouldn't wear pointed shoes or high heels? & 42.9 & 57.1 \\
Diabetics should always inspect their shoes before wearing them? & 50 & 50 \\
Diabetics should never wear shoes without socks? & 57.1 & 42.9 \\
Diabetics should never use hot water bottle on their feet? & 21.4 & 78.6 \\
Diabetics should never use their feet to & 42.9 & 57.1 \\
check the temperature of an object? & & \\
Diabetics should have their feet examined & 57.1 & 42.9 \\
by a doctor at least once a year? & 42.9 & 57.1 \\
\hline $\begin{array}{c}\text { Have you received any foot care education previously? } \\
\text { M }\end{array}$ & &
\end{tabular}

\section{Discussion}

This study shows the abysmally poor foot care knowledge among diabetic amputees. This is worrisome for two reasons. First, the incidence of diabetes is rising and hence the incidence of complications including LEA is also expected to rise concomitantly [6]. Secondly, unilateral LEA places the contralateral limb at greater risk of re-amputation [1] [5]. Thirdly, these patients have been to a healthcare facility where it is generally presumed that education about foot care will be communicated to them before discharge. Some authors have cited the busy clinic duty schedule of the doctors as part of the reasons for this poor communication [8]. In our hospital it may be worse, as the patient to doctor ratio is quite high, which may leave the doctor with little time to spend with each patient. Other studies have shown poor knowledge among diabetics with and without foot ulcers [4] [5] [9].

While most of the patients know that diabetics are at high risk of foot ulcers and amputations (78.6\%), only 50\% know they should inspect their feet daily. Fifty-seven percent know they should see a doctor to examine their feet at least once per year. The poorest answers were that of keeping in-between the toes dry and never to use hot water bottle on their feet. This poor knowledge despite being aware of the risk of amputation in diabetics is likely a reflection of lack of proper education regarding foot care practices.

The commonest cause of foot trauma in diabetics is inappropriate foot wear [5]. This, in conjunction with foot deformities [9], common in diabetics, can 
lead to callosities and ulcerations. Questions regarding foot wear showed that about half of them have poor knowledge in this regard. Half of the patients know they should inspect their feet daily, while a little more than half know that a professional should inspect at least once per year. This proportion is lower than that found by Muhammad et al. (70\%) [8].

Only duration of diabetes and educational level showed a significant association with foot care knowledge. It appears that the long standing diabetic might have had some time to receive some information about foot care. Most studies showed that educational level is associated with a higher level of foot care knowledge [5] [7] [9] [10] [11]. Only one study showed that educational level does not significantly affect foot care knowledge [8]. Age, gender, and prior amputation did not affect foot care knowledge. This agrees with other studies [8] [9].

Peripheral neuropathy is responsible for about $60 \%$ of diabetic foot ulcerations [9]. In our study, $71 \%$ of patients have peripheral neuropathy in the contralateral limb. With the loss of protective sensation and inability to sense micro-trauma, it becomes more imperative to emphasize regular foot care practices to this vulnerable group of patients. This will enable them to identify any foot lesion at the earliest stage.

\section{Conclusions and Recommendations}

It is clear that health care providers should put more effort in educating diabetic amputees on the risk factors for foot ulcerations, particularly on regular foot exam and appropriate foot wear. Giving them instructional leaflets before they are discharged from the hospitals will further cement such information in their memory. Such leaflets should be in plain and simple, easy to understand language.

Establishment of diabetic foot clinics, where periodic assessment of these patients will be undertaken cannot be over-emphasized. Such clinics have been shown to reduce the incidence of amputations [5] [11]. Other measures such as mass campaigns and radio jingles have been shown to be effective in communicable diseases such as HIV/AIDS [5]. Since the incidence of diabetes is increasing [7], and the burden of diabetic foot disease is expected to follow suit, it becomes necessary to sensitize the public about it.

\section{Limitations}

Multicenter study to increase the number of participants would be more desirable.

The study only looked at knowledge and did not study practice. Knowledge does not automatically translate to practice and such assumption must not be made based on this study.

\section{References}

[1] Carrington, A.L., Abbott, C.A., Griffiths, J., Jackson, N., Johnson, S.R., Kulkarni, J., 
et al. (2001) A Foot Care Program for Diabetic Unilateral Lower-Limb Amputees. Diabetes Care, 24, 216-221. https://doi.org/10.2337/diacare.24.2.216

[2] Frederiks, J.P. and Visagie, S. (2013) The Rehabilitation Programme and Functional Outcomes of Persons with Lower Limb Amputations at a Primary Level Rehabilitation Centre. South African Journal of Occupational Therapy, 43, 18-28.

[3] Hambleton, I.R., Jonnalagadda, R., Davis, C.R., Fraser, H.S., Chaturvedi, N. and Hennis, A.J. (2009) All-Cause Mortality after Diabetes-Related Amputation in Barbados. Diabetes Care, 32, 306-307. https://doi.org/10.2337/dc08-1504

[4] Nongmaithem, M., Bawa, A.P.S., Pithwa, A.K., Bhatia, S.K., Singh, G. and Gooptu, S. (2016) A Study of Risk Factors and Foot Care Behavior among Diabetics. Journal of Family Medicine and Primary Care, 5, 399-403.

https://doi.org/10.4103/2249-4863.192340

[5] Ikpeme, I.A., Udosen, A.M., Ngim, N.E., Ikpeme, A.A., Amah, P., Bello, S., et al. (2010) Footcare Practices among Nigerian Diabetic Patients Presenting with Foot Gangrene. African Journal of Diabetes Medicine, 18, 10.

[6] Desalu, O.O., Salawu, F.K., Jimoh, A.K., Adekoya, A.O., Busari, O.A. and Olokoba, A.B. (2011) Diabetic Foot Care: Self Reported Knowledge and Practice among Patients Attending Three Tertiary Hospital in Nigeria. Ghana Medical Journal, 45, 60-65. https://doi.org/10.4314/gmj.v45i2.68930

[7] Goie, T.T. and Naidoo, M. (2017) Awareness of Diabetic Foot Disease amongst Patients with Type 2 Diabetes Mellitus Attending the Chronic Outpatients Department at a Regional Hospital in Durban, South Africa. African Journal of Primary Health Care \& Family Medicine, 8, 1170. http://www.ncbi.nlm.nih.gov/pmc/articles/PMC5125263/

[8] Muhammad-Lutfi, A., Zaraihah, M. and Anuar-Ramdhan, I. (2014) Knowledge and Practice of Diabetic Foot Care in an In-Patient Setting at a Tertiary Medical Center. Malaysian Orthopaedic Journal, 8, 22-26. https://doi.org/10.5704/MOJ.1411.005

[9] Dahiru, I.L., Amaefule, K.E., Okpe, I.O., Ibrahim, A. and Muazu, S.B. (2016) An Overview of Diabetic Foot Disease. Nigerian Journal of Basic and Clinical Sciences, 13, 1. https://doi.org/10.4103/0331-8540.176206

[10] Saurabh, S., Sarkar, S., Selvaraj, K., Kar, S.S., Kumar, S.G. and Roy, G. (2014) Effectiveness of Foot Care Education among People with Type 2 Diabetes in Rural Puducherry, India. Indian Journal of Endocrinology and Metabolism, 18, 106-110. https://doi.org/10.4103/2230-8210.126587

[11] Ren, M., Yang, C., Lin, D.Z., Xiao, H.S., Mai, L.F., Guo, Y.C., et al. (2014) Effect of Intensive Nursing Education on the Prevention of Diabetic Foot Ulceration among Patients with High-Risk Diabetic Foot: A Follow-Up Analysis. Diabetes Technology \& Therapeutics, 16, 576-581. https://doi.org/10.1089/dia.2014.0004 